TRANSACTIONS OF THE

AMERICAN MATHEMATICAL SOCIETY

Volume 357, Number 12, Pages 4719-4737

S 0002-9947(05)04029-8

Article electronically published on June 29, 2005

\title{
$k$-HYPONORMALITY OF FINITE RANK PERTURBATIONS OF UNILATERAL WEIGHTED SHIFTS
}

\author{
RAÚL E. CURTO AND WOO YOUNG LEE
}

\begin{abstract}
In this paper we explore finite rank perturbations of unilateral weighted shifts $W_{\alpha}$. First, we prove that the subnormality of $W_{\alpha}$ is never stable under nonzero finite rank perturbations unless the perturbation occurs at the zeroth weight. Second, we establish that 2-hyponormality implies positive quadratic hyponormality, in the sense that the Maclaurin coefficients of $D_{n}(s):=\operatorname{det} P_{n}\left[\left(W_{\alpha}+s W_{\alpha}^{2}\right)^{*}, W_{\alpha}+s W_{\alpha}^{2}\right] P_{n}$ are nonnegative, for every $n \geq 0$, where $P_{n}$ denotes the orthogonal projection onto the basis vectors $\left\{e_{0}, \cdots, e_{n}\right\}$. Finally, for $\alpha$ strictly increasing and $W_{\alpha}$ 2-hyponormal, we show that for a small finite-rank perturbation $\alpha^{\prime}$ of $\alpha$, the shift $W_{\alpha^{\prime}}$ remains quadratically hyponormal.
\end{abstract}

\section{INTRODUCTION}

Let $\mathcal{H}$ and $\mathcal{K}$ be complex Hilbert spaces, let $\mathcal{L}(\mathcal{H}, \mathcal{K})$ be the set of bounded linear operators from $\mathcal{H}$ to $\mathcal{K}$ and write $\mathcal{L}(\mathcal{H}):=\mathcal{L}(\mathcal{H}, \mathcal{H})$. An operator $T \in \mathcal{L}(\mathcal{H})$ is said to be normal if $T^{*} T=T T^{*}$, hyponormal if $T^{*} T \geq T T^{*}$, and subnormal if $T=\left.N\right|_{\mathcal{H}}$, where $N$ is normal on some Hilbert space $\mathcal{K} \supseteq \mathcal{H}$. If $T$ is subnormal, then $T$ is also hyponormal. Recall that given a bounded sequence of positive numbers $\alpha: \alpha_{0}, \alpha_{1}, \cdots$ (called weights), the (unilateral) weighted shift $W_{\alpha}$ associated with $\alpha$ is the operator on $\ell^{2}\left(\mathbb{Z}_{+}\right)$defined by $W_{\alpha} e_{n}:=\alpha_{n} e_{n+1}$ for all $n \geq 0$, where $\left\{e_{n}\right\}_{n=0}^{\infty}$ is the canonical orthonormal basis for $\ell^{2}$. It is straightforward to check that $W_{\alpha}$ can never be normal, and that $W_{\alpha}$ is hyponormal if and only if $\alpha_{n} \leq \alpha_{n+1}$ for all $n \geq 0$. The Bram-Halmos criterion for subnormality states that an operator $T$ is subnormal if and only if

$$
\sum_{i, j}\left(T^{i} x_{j}, T^{j} x_{i}\right) \geq 0
$$

Received by the editors December 10, 1999 and, in revised form, December 31, 2001.

2000 Mathematics Subject Classification. Primary 47B20, 47B35, 47B37; Secondary 47-04, 47A20, 47A57.

Key words and phrases. Weighted shifts, perturbations, subnormal, $k$-hyponormal, weakly $k$-hyponormal.

The work of the first-named author was partially supported by NSF research grants DMS9800931 and DMS-0099357.

The work of the second-named author was partially supported by a grant (R14-2003-006-010010) from the Korea Science and Engineering Foundation. 
for all finite collections $x_{0}, x_{1}, \cdots, x_{k} \in \mathcal{H}$ ([2], 4, II.1.9]). It is easy to see that this is equivalent to the following positivity test:

$$
\left(\begin{array}{cccc}
I & T^{*} & \ldots & T^{* k} \\
T & T^{*} T & \ldots & T^{* k} T \\
\vdots & \vdots & \ddots & \vdots \\
T^{k} & T^{*} T^{k} & \ldots & T^{* k} T^{k}
\end{array}\right) \geq 0 \quad \text { (for all } k \geq 1 \text { ). }
$$

Condition (1.1) provides a measure of the gap between hyponormality and subnormality. In fact, the positivity condition (1.1) for $k=1$ is equivalent to the hyponormality of $T$, while subnormality requires the validity of (1.1) for all $k$. Let $[A, B]:=A B-B A$ denote the commutator of two operators $A$ and $B$, and define $T$ to be $k$-hyponormal whenever the $k \times k$ operator matrix

$$
M_{k}(T):=\left(\left[T^{* j}, T^{i}\right]\right)_{i, j=1}^{k}
$$

is positive. An application of the Choleski algorithm for operator matrices shows that the positivity of $(1.2)$ is equivalent to the positivity of the $(k+1) \times(k+1)$ operator matrix in (1.1); the Bram-Halmos criterion can then be rephrased to say that $T$ is subnormal if and only if $T$ is $k$-hyponormal for every $k \geq 1$ ([16]).

Recall ([1, [16], [5]) that $T \in \mathcal{L}(\mathcal{H})$ is said to be weakly $k$-hyponormal if

$$
L S\left(T, T^{2}, \cdots, T^{k}\right):=\left\{\sum_{j=1}^{k} \alpha_{j} T^{j}: \alpha=\left(\alpha_{1}, \cdots, \alpha_{k}\right) \in \mathbb{C}^{k}\right\}
$$

consists entirely of hyponormal operators, or equivalently, $M_{k}(T)$ is weakly positive, i.e. $(16)$,

$$
\left(M_{k}(T)\left(\begin{array}{c}
\lambda_{0} x \\
\vdots \\
\lambda_{k} x
\end{array}\right),\left(\begin{array}{c}
\lambda_{0} x \\
\vdots \\
\lambda_{k} x
\end{array}\right)\right) \geq 0 \quad \text { for } x \in \mathcal{H} \text { and } \lambda_{0}, \cdots, \lambda_{k} \in \mathbb{C} .
$$

If $k=2$, then $T$ is said to be quadratically hyponormal, and if $k=3$, then $T$ is said to be cubically hyponormal. Similarly, $T \in \mathcal{L}(\mathcal{H})$ is said to be polynomially hyponormal if $p(T)$ is hyponormal for every polynomial $p \in \mathbb{C}[z]$. It is known that $k$-hyponormal $\Rightarrow$ weakly $k$-hyponormal, but the converse is not true in general.

The classes of (weakly) $k$-hyponormal operators have been studied in an attempt to bridge the gap between subnormality and hyponormality $(7], 8], 10], 11,[12$, 14, [16, 19], 22]). The study of this gap has been only partially successful. For example, such a gap is not yet well described for Toeplitz operators on the Hardy space of the unit circle; in fact, even subnormality for Toeplitz operators has not been characterized (cf. 20], 6]). For weighted shifts, positive results appear in [7] and 12, although no concrete example of a weighted shift which is polynomially hyponormal and not subnormal has yet been found (the existence of such weighted shifts was established in [17] and [18]).

In the present paper we renew our efforts to help describe the above-mentioned gap between subnormality and hyponormality, with particular emphasis on polynomial hyponormality. We focus on the class of unilateral weighted shifts, and initiate a study of how the above-mentioned notions behave under finite perturbations of the weight sequence. We first obtain the following three concrete results. 
(i) the subnormality of $W_{\alpha}$ is never stable under nonzero finite rank perturbations unless the perturbation is confined to the zeroth weight (Theorem 2.1);

(ii) 2-hyponormality implies positive quadratic hyponormality, in the sense that the Maclaurin coefficients of $D_{n}(s):=\operatorname{det} P_{n}\left[\left(W_{\alpha}+s W_{\alpha}^{2}\right)^{*}, W_{\alpha}+s W_{\alpha}^{2}\right] P_{n}$ are nonnegative, for every $n \geq 0$, where $P_{n}$ denotes the orthogonal projection onto the basis vectors $\left\{e_{0}, \cdots, e_{n}\right\}$ (Theorem 2.2 ); and

(iii) if $\alpha$ is strictly increasing and $W_{\alpha}$ is 2-hyponormal, then for $\alpha^{\prime}$ a small perturbation of $\alpha$, the shift $W_{\alpha^{\prime}}$ remains positively quadratically hyponormal (Theorem $2.3)$.

Along the way we establish two related results, each of independent interest:

(iv) an integrality criterion for a subnormal weighted shift to have an $n$-step subnormal extension (Theorem 6.1); and

(v) a proof that the sets of $k$-hyponormal and weakly $k$-hyponormal operators are closed in the strong operator topology (Proposition 6.7).

\section{Statement of main Results}

C. Berger's characterization of subnormality for unilateral weighted shifts (cf. [21, 4, III.8.16]) states that $W_{\alpha}$ is subnormal if and only if there exists a Borel probability measure $\mu$ (the so-called Berger measure of $W_{\alpha}$ ) supported in $\left[0,\left\|W_{\alpha}\right\|^{2}\right]$, with $\left\|W_{\alpha}\right\|^{2} \in \operatorname{supp} \mu$, such that

$$
\gamma_{n}=\int t^{n} d \mu(t) \quad \text { for all } n \geq 0 .
$$

Given an initial segment of weights $\alpha: \alpha_{0}, \cdots, \alpha_{m}$, the sequence $\hat{\alpha} \in \ell^{\infty}\left(\mathbb{Z}_{+}\right)$such that $\hat{\alpha}_{i}=\alpha_{i}(i=0, \cdots, m)$ is said to be recursively generated by $\alpha$ if there exist $r \geq 1$ and $\varphi_{0}, \cdots, \varphi_{r-1} \in \mathbb{R}$ such that

$$
\gamma_{n+r}=\varphi_{0} \gamma_{n}+\cdots+\varphi_{r-1} \gamma_{n+r-1} \quad(\text { for all } n \geq 0)
$$

where $\gamma_{0}:=1, \gamma_{n}:=\alpha_{0}^{2} \cdots \alpha_{n-1}^{2}(n \geq 1)$. In this case $W_{\hat{\alpha}}$ with weights $\hat{\alpha}$ is said to be recursively generated. If we let

$$
g(t):=t^{r}-\left(\varphi_{r-1} t^{r-1}+\cdots+\varphi_{0}\right)
$$

then $g$ has $r$ distinct real roots $0 \leq s_{0}<\cdots<s_{r-1}$ ([11, Theorem 3.9]). Let

$$
V:=\left(\begin{array}{cccc}
1 & 1 & \ldots & 1 \\
s_{0} & s_{1} & \ldots & s_{r-1} \\
\vdots & \vdots & & \vdots \\
s_{0}^{r-1} & s_{1}^{r-1} & \ldots & s_{r-1}^{r-1}
\end{array}\right)
$$

and let

$$
\left(\begin{array}{c}
\rho_{0} \\
\vdots \\
\rho_{r-1}
\end{array}\right):=V^{-1}\left(\begin{array}{c}
\gamma_{0} \\
\vdots \\
\gamma_{r-1}
\end{array}\right)
$$

If the associated recursively generated weighted shift $W_{\hat{\alpha}}$ is subnormal, then its Berger measure is of the form

$$
\mu:=\rho_{0} \delta_{s_{0}}+\cdots+\rho_{r-1} \delta_{r-1}
$$


For example, given $\alpha_{0}<\alpha_{1}<\alpha_{2}, W_{\left(\alpha_{0}, \alpha_{1}, \alpha_{2}\right)^{\wedge}}$ is the recursive weighted shift whose weights are calculated according to the recursive relation

$$
\alpha_{n+1}^{2}=\varphi_{1}+\varphi_{0} \frac{1}{\alpha_{n}^{2}}
$$

where

$$
\varphi_{0}=-\frac{\alpha_{0}^{2} \alpha_{1}^{2}\left(\alpha_{2}^{2}-\alpha_{1}^{2}\right)}{\alpha_{1}^{2}-\alpha_{0}^{2}} \quad \text { and } \quad \varphi_{1}=\frac{\alpha_{1}^{2}\left(\alpha_{2}^{2}-\alpha_{0}^{2}\right)}{\alpha_{1}^{2}-\alpha_{0}^{2}} .
$$

In this case, $W_{\left(\alpha_{0}, \alpha_{1}, \alpha_{2}\right)^{\wedge}}$ is subnormal with 2-atomic Berger measure. Let $W_{x\left(\alpha_{0}, \alpha_{1}, \alpha_{2}\right) \wedge}$ denote the weighted shift whose weight sequence consists of the initial weight $x$ followed by the weight sequence of $W_{\left(\alpha_{0}, \alpha_{1}, \alpha_{2}\right)^{\wedge}}$.

By the Density Theorem ([11, Theorem 4.2 and Corollary 4.3]), we know that if $W_{\alpha}$ is a subnormal weighted shift with weights $\alpha=\left\{\alpha_{n}\right\}$ and $\epsilon>0$, then there exists a nonzero compact operator $K$ with $\|K\|<\epsilon$ such that $W_{\alpha}+K$ is a recursively generated subnormal weighted shift; in fact $W_{\alpha}+K=W_{\widehat{\alpha^{(m)}}}$ for some $m \geq 1$, where $\alpha^{(m)}: \alpha_{0}, \cdots, \alpha_{m}$. The following result shows that $K$ cannot generally be taken to be of finite rank.

Theorem 2.1 (Finite Rank Perturbations of Subnormal Shifts). If $W_{\alpha}$ is a subnormal weighted shift, then there exists no nonzero finite rank operator $F\left(\neq c P_{\left\{e_{0}\right\}}\right)$ such that $W_{\alpha}+F$ is a subnormal weighted shift. Concretely, suppose $W_{\alpha}$ is a subnormal weighted shift with weight sequence $\alpha=\left\{\alpha_{n}\right\}_{n=0}^{\infty}$ and assume $\alpha^{\prime}=\left\{\alpha_{n}^{\prime}\right\}$ is a nonzero perturbation of $\alpha$ in a finite number of weights except the initial weight. Then $W_{\alpha^{\prime}}$ is not subnormal.

We next consider the self-commutator $\left[\left(W_{\alpha}+s W_{\alpha}^{2}\right)^{*}, W_{\alpha}+s W_{\alpha}^{2}\right]$. Let $W_{\alpha}$ be a hyponormal weighted shift. For $s \in \mathbb{C}$, we write

$$
D(s):=\left[\left(W_{\alpha}+s W_{\alpha}^{2}\right)^{*}, W_{\alpha}+s W_{\alpha}^{2}\right]
$$

and we let

$$
\begin{aligned}
D_{n}(s) & :=P_{n}\left[\left(W_{\alpha}+s W_{\alpha}^{2}\right)^{*}, W_{\alpha}+s W_{\alpha}^{2}\right] P_{n} \\
& =\left(\begin{array}{cccccc}
q_{0} & \bar{r}_{0} & 0 & \ldots & 0 & 0 \\
r_{0} & q_{1} & \bar{r}_{1} & \ldots & 0 & 0 \\
0 & r_{1} & q_{2} & \ldots & 0 & 0 \\
\vdots & \vdots & \vdots & \ddots & \vdots & \vdots \\
0 & 0 & 0 & \ldots & q_{n-1} & \bar{r}_{n-1} \\
0 & 0 & 0 & \ldots & r_{n-1} & q_{n}
\end{array}\right),
\end{aligned}
$$

where $P_{n}$ is the orthogonal projection onto the subspace generated by $\left\{e_{0}, \cdots, e_{n}\right\}$,

$$
\left\{\begin{array}{l}
q_{n}:=u_{n}+|s|^{2} v_{n} \\
r_{n}:=s \sqrt{w_{n}} \\
u_{n}:=\alpha_{n}^{2}-\alpha_{n-1}^{2} \\
v_{n}:=\alpha_{n}^{2} \alpha_{n+1}^{2}-\alpha_{n-1}^{2} \alpha_{n-2}^{2}, \\
w_{n}:=\alpha_{n}^{2}\left(\alpha_{n+1}^{2}-\alpha_{n-1}^{2}\right)^{2}
\end{array}\right.
$$

and, for notational convenience, $\alpha_{-2}=\alpha_{-1}=0$. Clearly, $W_{\alpha}$ is quadratically hyponormal if and only if $D_{n}(s) \geq 0$ for all $s \in \mathbb{C}$ and all $n \geq 0$. Let $d_{n}(\cdot):=$ 
$\operatorname{det}\left(D_{n}(\cdot)\right)$. Then $d_{n}$ satisfies the following 2-step recursive formula:

$$
d_{0}=q_{0}, \quad d_{1}=q_{0} q_{1}-\left|r_{0}\right|^{2}, \quad d_{n+2}=q_{n+2} d_{n+1}-\left|r_{n+1}\right|^{2} d_{n} .
$$

If we let $t:=|s|^{2}$, we observe that $d_{n}$ is a polynomial in $t$ of degree $n+1$, and if we write $d_{n} \equiv \sum_{i=0}^{n+1} c(n, i) t^{i}$, then the coefficients $c(n, i)$ satisfy a double-indexed recursive formula, namely

$$
\begin{aligned}
& c(n+2, i)=u_{n+2} c(n+1, i)+v_{n+2} c(n+1, i-1)-w_{n+1} c(n, i-1), \\
& c(n, 0)=u_{0} \cdots u_{n}, \quad c(n, n+1)=v_{0} \cdots v_{n}, \quad c(1,1)=u_{1} v_{0}+v_{1} u_{0}-w_{0}
\end{aligned}
$$

$(n \geq 0, i \geq 1)$. We say that $W_{\alpha}$ is positively quadratically hyponormal if $c(n, i) \geq 0$ for every $n \geq 0,0 \leq i \leq n+1$ (cf. [9]). Evidently, positively quadratically hyponormal $\Longrightarrow$ quadratically hyponormal. The converse, however, is not true in general (cf. [3]).

The following theorem establishes a useful relation between 2-hyponormality and positive quadratic hyponormality.

Theorem 2.2. Let $\alpha \equiv\left\{\alpha_{n}\right\}_{n=0}^{\infty}$ be a weight sequence and assume that $W_{\alpha}$ is 2-hyponormal. Then $W_{\alpha}$ is positively quadratically hyponormal. More precisely, if $W_{\alpha}$ is 2-hyponormal, then

$$
c(n, i) \geq v_{0} \cdots v_{i-1} u_{i} \cdots u_{n} \quad(n \geq 0,0 \leq i \leq n+1) .
$$

In particular, if $\alpha$ is strictly increasing and $W_{\alpha}$ is 2-hyponormal, then the Maclaurin coefficients of $d_{n}(t)$ are positive for all $n \geq 0$.

If $W_{\alpha}$ is a weighted shift with weight sequence $\alpha=\left\{\alpha_{n}\right\}_{n=0}^{\infty}$, then the moments of $W_{\alpha}$ are usually defined by $\beta_{0}:=1, \beta_{n+1}:=\alpha_{n} \beta_{n}(n \geq 0)$ [23]; however, we prefer to reserve this term for the sequence $\gamma_{n}:=\beta_{n}^{2}(n \geq 0)$. A criterion for $k$-hyponormality can be given in terms of these moments $([\overline{7}$, Theorem 4$])$ : if we build a $(k+1) \times(k+1)$ Hankel matrix $A(n ; k)$ by

$$
A(n ; k):=\left(\begin{array}{cccc}
\gamma_{n} & \gamma_{n+1} & \ldots & \gamma_{n+k} \\
\gamma_{n+1} & \gamma_{n+2} & \ldots & \gamma_{n+k+1} \\
\vdots & \vdots & & \vdots \\
\gamma_{n+k} & \gamma_{n+k+1} & \ldots & \gamma_{n+2 k}
\end{array}\right) \quad(n \geq 0)
$$

then

$$
W_{\alpha} \text { is } k \text {-hyponormal } \Longleftrightarrow A(n ; k) \geq 0 \quad(n \geq 0) \text {. }
$$

In particular, for $\alpha$ strictly increasing, $W_{\alpha}$ is 2-hyponormal if and only if

$$
\operatorname{det}\left(\begin{array}{ccc}
\gamma_{n} & \gamma_{n+1} & \gamma_{n+2} \\
\gamma_{n+1} & \gamma_{n+2} & \gamma_{n+3} \\
\gamma_{n+2} & \gamma_{n+3} & \gamma_{n+4}
\end{array}\right) \geq 0 \quad(n \geq 0)
$$

One might conjecture that if $W_{\alpha}$ is a $k$-hyponormal weighted shift whose weight sequence is strictly increasing, then $W_{\alpha}$ remains weakly $k$-hyponormal under a small perturbation of the weight sequence. We will show below that this is true for $k=2$ (Theorem 2.3).

In [12, Theorem 4.3], it was shown that the gap between 2-hyponormality and quadratic hyponormality can be detected by unilateral shifts with a weight sequence $\alpha: \sqrt{x},(\sqrt{a}, \sqrt{b}, \sqrt{c})^{\wedge}$. In particular, there exists a maximum value $H_{2} \equiv H_{2}(a, b, c)$ 
of $x$ that makes $W_{\sqrt{x},(\sqrt{a}, \sqrt{b}, \sqrt{c})^{\wedge}}$ 2-hyponormal; $H_{2}$ is called the modulus of 2hyponormality (cf. [12]). Any value of $x>H_{2}$ yields a non-2-hyponormal weighted shift. However, if $x-H_{2}$ is small enough, $W_{\sqrt{x},(\sqrt{a}, \sqrt{b}, \sqrt{c})^{\wedge}}$ is still quadratically hyponormal. The following theorem shows that, more generally, for finite rank perturbations of weighted shifts with strictly increasing weight sequences, there always exists a gap between 2-hyponormality and quadratic hyponormality.

Theorem 2.3 (Finite Rank Perturbations of 2-Hyponormal Shifts). Let $\alpha=$ $\left\{\alpha_{n}\right\}_{n=0}^{\infty}$ be a strictly increasing weight sequence. If $W_{\alpha}$ is 2-hyponormal, then $W_{\alpha}$ remains positively quadratically hyponormal under a small nonzero finite rank perturbation of $\alpha$.

\section{Proof of Theorem 2.1}

Proof of Theorem 2.1. It suffices to show that if $T$ is a weighted shift whose restriction to $\bigvee\left\{e_{n}, e_{n+1}, \cdots\right\}(n \geq 2)$ is subnormal, then there is at most one $\alpha_{n-1}$ for which $T$ is subnormal.

Let $W:=\left.T\right|_{\bigvee\left\{e_{n-1}, e_{n}, e_{n+1}, \cdots\right\}}$ and $S:=\left.T\right|_{\bigvee\left\{e_{n}, e_{n+1}, \cdots\right\}}$, where $n \geq 2$. Then $W$ and $S$ have weights $\alpha_{k}(W):=\alpha_{k+n-1}$ and $\alpha_{k}(S):=\alpha_{k+n}(k \geq 0)$. Thus the corresponding moments are related by the equation

$$
\gamma_{k}(S)=\alpha_{n}^{2} \cdots \alpha_{n+k-1}^{2}=\frac{\gamma_{k+1}(W)}{\alpha_{n-1}^{2}}
$$

We now adapt the proof of [7, Proposition 8]. Suppose $S$ is subnormal with associated Berger measure $\mu$. Then $\gamma_{k}(S)=\int_{0}^{\|T\|^{2}} t^{k} d \mu$. Thus $W$ is subnormal if and only if there exists a probability measure $\nu$ on $\left[0,\|T\|^{2}\right]$ such that

$$
\frac{1}{\alpha_{n-1}^{2}} \int_{0}^{\|T\|^{2}} t^{k+1} d \nu(t)=\int_{0}^{\|T\|^{2}} t^{k} d \mu(t) \quad \text { for all } k \geq 0,
$$

which readily implies that $t d \nu=\alpha_{n-1}^{2} d \mu$. Thus $W$ is subnormal if and only if the formula

$$
d \nu:=\lambda \cdot \delta_{0}+\frac{\alpha_{n-1}^{2}}{t} d \mu
$$

defines a probability measure for some $\lambda \geq 0$, where $\delta_{0}$ is the point mass at the origin. In particular $\frac{1}{t} \in L^{1}(\mu)$ and $\mu(\{0\})=0$ whenever $W$ is subnormal. If we repeat the above argument for $W$ and $V:=\left.T\right|_{\bigvee\left\{e_{n-2}, e_{n-1}, \cdots\right\}}$, then we should have that $\nu(\{0\})=0$ whenever $V$ is subnormal. Therefore we can conclude that if $V$ is subnormal, then $\lambda=0$, and hence

$$
d \nu=\frac{\alpha_{n-1}^{2}}{t} d \mu
$$

Thus we have

$$
1=\int_{0}^{\|T\|^{2}} d \nu(t)=\alpha_{n-1}^{2} \int_{0}^{\|T\|^{2}} \frac{1}{t} d \mu(t)
$$

so that

$$
\alpha_{n-1}^{2}=\left(\int_{0}^{\|T\|^{2}} \frac{1}{t} d \mu(t)\right)^{-1}
$$


which implies that $\alpha_{n-1}$ is determined uniquely by $\left\{\alpha_{n}, \alpha_{n+1}, \cdots\right\}$ whenever $T$ is subnormal. This completes the proof.

Theorem 2.1 says that a nonzero finite rank perturbation of a subnormal shift is never subnormal unless the perturbation occurs at the initial weight. However, this is not the case for $k$-hyponormality. To see this we use a close relative of the Bergman shift $B_{+}$(whose weights are given by $\alpha=\left\{\sqrt{\frac{n+1}{n+2}}\right\}_{n=0}^{\infty}$ ); it is well known that $B_{+}$is subnormal.

Example 3.1. For $x>0$, let $T_{x}$ be the weighted shift whose weights are given by

$$
\alpha_{0}:=\sqrt{\frac{1}{2}}, \quad \alpha_{1}:=\sqrt{x}, \text { and } \alpha_{n}:=\sqrt{\frac{n+1}{n+2}}(n \geq 2)
$$

Then we have:

(i) $T_{x}$ is subnormal $\Longleftrightarrow x=\frac{2}{3}$;

(ii) $T_{x}$ is 2-hyponormal $\Longleftrightarrow \frac{63-\sqrt{129}}{80} \leq x \leq \frac{24}{35}$.

Proof. Assertion (i) follows from Theorem 2.1. For assertion (ii) we use (2.12): $T_{x}$ is 2-hyponormal if and only if

$$
\operatorname{det}\left(\begin{array}{ccc}
1 & \frac{1}{2} & \frac{1}{2} x \\
\frac{1}{2} & \frac{1}{2} x & \frac{3}{8} x \\
\frac{1}{2} x & \frac{3}{8} x & \frac{3}{10} x
\end{array}\right) \geq 0 \quad \text { and } \operatorname{det}\left(\begin{array}{ccc}
\frac{1}{2} & \frac{1}{2} x & \frac{3}{8} x \\
\frac{1}{2} x & \frac{3}{8} x & \frac{3}{10} x \\
\frac{3}{8} x & \frac{3}{10} x & \frac{1}{4} x
\end{array}\right) \geq 0,
$$

or equivalently, $\frac{63-\sqrt{129}}{80} \leq x \leq \frac{24}{35}$.

For perturbations of recursive subnormal shifts of the form $W_{(\sqrt{a}, \sqrt{b}, \sqrt{c})^{\wedge}}$, subnormality and 2-hyponormality coincide.

Theorem 3.2. Let $\alpha=\left\{\alpha_{n}\right\}_{n=0}^{\infty}$ be recursively generated by $\sqrt{a}, \sqrt{b}, \sqrt{c}$. If $T_{x}$ is the weighted shift whose weights are given by $\alpha_{x}: \alpha_{0}, \cdots, \alpha_{j-1}, \sqrt{x}, \alpha_{j+1}, \cdots$, then we have

$$
T_{x} \text { is subnormal } \Longleftrightarrow T_{x} \text { is 2-hyponormal } \Longleftrightarrow \begin{cases}x=\alpha_{j}^{2} & \text { if } j \geq 1 ; \\ x \leq a & \text { if } j=0 .\end{cases}
$$

Proof. Since $\alpha$ is recursively generated by $\sqrt{a}, \sqrt{b}, \sqrt{c}$, we have that $\alpha_{0}^{2}=a, \alpha_{1}^{2}=$ $b, \alpha_{2}^{2}=c$,

$$
\begin{aligned}
& \alpha_{3}^{2}=\frac{b\left(c^{2}-2 a c+a b\right)}{c(b-a)}, \text { and } \\
& \alpha_{4}^{2}=\frac{b c^{3}-4 a b c^{2}+2 a b^{2} c+a^{2} b c-a^{2} b^{2}+a^{2} c^{2}}{(b-a)\left(c^{2}-2 a c+a b\right)} .
\end{aligned}
$$

Case $1(j=0)$ : It is evident that $T_{x}$ is subnormal if and only if $x \leq a$. For 2-hyponormality observe by (2.12) that $T_{x}$ is 2-hyponormal if and only if

$$
\operatorname{det}\left(\begin{array}{ccc}
1 & x & b x \\
x & b x & b c x \\
b x & b c x & \alpha_{3}^{2} b c x
\end{array}\right) \geq 0
$$

or equivalently, $x \leq a$. 
Case $2(j \geq 1)$ : Without loss of generality we may assume that $j=1$ and $a=1$. Thus $\alpha_{1}=\sqrt{x}$. Then by Theorem $2.1, T_{x}$ is subnormal if and only if $x=b$. On the other hand, by (2.12), $T_{x}$ is 2-hyponormal if and only if

$$
\operatorname{det}\left(\begin{array}{ccc}
1 & 1 & x \\
1 & x & c x \\
x & c x & \alpha_{3}^{2} c x
\end{array}\right) \geq 0 \quad \text { and } \operatorname{det}\left(\begin{array}{ccc}
1 & x & c x \\
x & c x & \alpha_{3}^{2} c x \\
c x & \alpha_{3}^{2} c x & \alpha_{3}^{2} \alpha_{4}^{2} c x
\end{array}\right) \geq 0
$$

Thus a direct calculation with the specific forms of $\alpha_{3}, \alpha_{4}$ given in (3.4) shows that $T_{x}$ is 2-hyponormal if and only if $(x-b)\left(x-\frac{b\left(c^{2}-2 c+b\right)}{b-1}\right) \leq 0$ and $x \leq b$. Since $b \leq \frac{b\left(c^{2}-2 c+b\right)}{b-1}$, it follows that $T_{x}$ is 2-hyponormal if and only if $x=b$. This completes the proof.

\section{Proof of Theorem 2.2}

With the notation in (2.6), we let

$$
p_{n}:=u_{n} v_{n+1}-w_{n} \quad(n \geq 0) .
$$

We then have:

Lemma 4.1. If $\alpha \equiv\left\{\alpha_{n}\right\}_{n=0}^{\infty}$ is a strictly increasing weight sequence, then the following statements are equivalent:

(i) $W_{\alpha}$ is 2-hyponormal;

(ii) $\alpha_{n+1}^{2}\left(u_{n+1}+u_{n+2}\right)^{2} \leq u_{n+1} v_{n+2} \quad(n \geq 0)$;

(iii) $\frac{\alpha_{n}^{2}}{\alpha_{n+2}^{2}} \frac{u_{n+2}}{u_{n+3}} \leq \frac{u_{n+1}}{u_{n+2}} \quad(n \geq 0)$;

(iv) $p_{n} \geq 0 \quad(n \geq 0)$.

Proof. This follows from a straightforward calculation.

Proof of Theorem 2.2. If $\alpha$ is not strictly increasing, then $\alpha$ is flat, by the argument of [7, Corollary 6], i.e., $\alpha_{0}=\alpha_{1}=\alpha_{2}=\cdots$. Then

$$
D_{n}(s)=\left(\begin{array}{cc}
\alpha_{0}^{2}+|s|^{2} \alpha_{0}^{4} & \bar{s} \alpha_{0}^{3} \\
s \alpha_{0}^{3} & |s|^{2} \alpha_{0}^{4}
\end{array}\right) \oplus 0_{\infty}
$$

(cf. (2.5)), so that (2.9) is evident. Thus we may assume that $\alpha$ is strictly increasing, so that $u_{n}>0, v_{n}>0$ and $w_{n}>0$ for all $n \geq 0$. Recall that if we write $d_{n}(t):=\sum_{i=0}^{n+1} c(n, i) t^{i}$, then the $c(n, i)$ 's satisfy the following recursive formulas (cf. $(2.8))$ :

$$
\begin{aligned}
c(n+2, i)= & u_{n+2} c(n+1, i)+v_{n+2} c(n+1, i-1) \\
& -w_{n+1} c(n, i-1) \quad(n \geq 0,1 \leq i \leq n) .
\end{aligned}
$$

Also, $c(n, n+1)=v_{0} \cdots v_{n}$ (again by $\left.(2.8)\right)$ and $p_{n}:=u_{n} v_{n+1}-w_{n} \geq 0(n \geq 0)$, by Lemma 4.1. A straightforward calculation shows that

$$
\begin{aligned}
d_{0}(t)= & u_{0}+v_{0} t \\
d_{1}(t)= & u_{0} u_{1}+\left(v_{0} u_{1}+p_{0}\right) t+v_{0} v_{1} t^{2} \\
d_{2}(t)= & u_{0} u_{1} u_{2}+\left(v_{0} u_{1} u_{2}+u_{0} p_{1}+u_{2} p_{0}\right) t \\
& +\left(v_{0} v_{1} u_{2}+v_{0} p_{1}+v_{2} p_{0}\right) t^{2}+v_{0} v_{1} v_{2} t^{3} .
\end{aligned}
$$

Evidently,

$$
c(n, i) \geq 0 \quad(0 \leq n \leq 2,0 \leq i \leq n+1)
$$


Define

$$
\beta(n, i):=c(n, i)-v_{0} \cdots v_{i-1} u_{i} \cdots u_{n} \quad(n \geq 1,1 \leq i \leq n) .
$$

For every $n \geq 1$, we now have

$$
c(n, i)= \begin{cases}u_{0} \cdots u_{n} \geq 0 & (i=0), \\ v_{0} \cdots v_{i-1} u_{i} \cdots u_{n}+\beta(n, i) & (1 \leq i \leq n), \\ v_{0} \cdots v_{n} \geq 0 & (i=n+1) .\end{cases}
$$

For notational convenience we let $\beta(n, 0):=0$ for every $n \geq 0$.

Claim 1. For $n \geq 1$,

$$
c(n, n) \geq u_{n} c(n-1, n) \geq 0 .
$$

Proof of Claim 1. We use mathematical induction. For $n=1$,

$$
c(1,1)=v_{0} u_{1}+p_{0} \geq u_{1} c(0,1) \geq 0,
$$

and

$$
\begin{aligned}
c(n+1, n+1) & =u_{n+1} c(n, n+1)+v_{n+1} c(n, n)-w_{n} c(n-1, n) \\
& \geq u_{n+1} c(n, n+1)+v_{n+1} u_{n} c(n-1, n)-w_{n} c(n-1, n)
\end{aligned}
$$

(by the inductive hypothesis)

$$
\begin{aligned}
& =u_{n+1} c(n, n+1)+p_{n} c(n-1, n) \\
& \geq u_{n+1} c(n, n+1),
\end{aligned}
$$

which proves Claim 1.

Claim 2. For $n \geq 2$,

$$
\beta(n, i) \geq u_{n} \beta(n-1, i) \geq 0 \quad(0 \leq i \leq n-1) .
$$

Proof of Claim 2. We use mathematical induction. If $n=2$ and $i=0$, this is trivial. Also,

$$
\beta(2,1)=u_{0} p_{1}+u_{2} p_{0}=u_{0} p_{1}+u_{2} \beta(1,1) \geq u_{2} \beta(1,1) \geq 0 .
$$

Assume that (4.7) holds. We shall prove that

$$
\beta(n+1, i) \geq u_{n+1} \beta(n, i) \geq 0 \quad(0 \leq i \leq n) .
$$

For,

$$
\begin{gathered}
\beta(n+1, i)+v_{0} \cdots v_{i-1} u_{i} \cdots u_{n+1}=c(n+1, i) \quad(\text { by }(4.2)) \\
=u_{n+1} c(n, i)+v_{n+1} c(n, i-1)-w_{n} c(n-1, i-1) \\
=u_{n+1}\left(\beta(n, i)+v_{0} \cdots v_{i-1} u_{i} \cdots u_{n}\right) \\
+v_{n+1}\left(\beta(n, i-1)+v_{0} \cdots v_{i-2} u_{i-1} \cdots u_{n}\right) \\
\quad-w_{n}\left(\beta(n-1, i-1)+v_{0} \cdots v_{i-2} u_{i-1} \cdots u_{n-1}\right),
\end{gathered}
$$


so that

$$
\begin{aligned}
\beta(n+1, i)= & u_{n+1} \beta(n, i)+v_{n+1} \beta(n, i-1)-w_{n} \beta(n-1, i-1) \\
& +v_{0} \cdots v_{i-2} u_{i-1} \cdots u_{n-1}\left(u_{n} v_{n+1}-w_{n}\right) \\
= & u_{n+1} \beta(n, i)+v_{n+1} \beta(n, i-1)-w_{n} \beta(n-1, i-1) \\
& +\left(v_{0} \cdots v_{i-2} u_{i-1} \cdots u_{n-1}\right) p_{n} \\
\geq & u_{n+1} \beta(n, i)+v_{n+1} u_{n} \beta(n-1, i-1)-w_{n} \beta(n-1, i-1)
\end{aligned}
$$

(by the inductive hypothesis and Lemma 4.1;

observe that $i-1 \leq n-1$, so (4.7) applies)

$$
\begin{aligned}
& =u_{n+1} \beta(n, i)+p_{n} \beta(n-1, i-1) \\
& \geq u_{n+1} \beta(n, i),
\end{aligned}
$$

which proves Claim 2.

By Claim 2 and (4.5), we can see that $c(n, i) \geq 0$ for all $n \geq 0$ and $1 \leq i \leq n-1$. Therefore (4.4), (4.5), Claim 1 and Claim 2 imply

$$
c(n, i) \geq v_{0} \cdots v_{i-1} u_{i} \cdots u_{n} \quad(n \geq 0,0 \leq i \leq n+1) .
$$

This completes the proof.

\section{Proof of Theorem 2.3}

To prove Theorem 2.3 we need:

Lemma 5.1 ([15, Lemma 2.3]). Let $\alpha \equiv\left\{\alpha_{n}\right\}_{n=0}^{\infty}$ be a strictly increasing weight sequence. If $W_{\alpha}$ is 2-hyponormal, then the sequence of quotients

$$
\Theta_{n}:=\frac{u_{n+1}}{u_{n+2}} \quad(n \geq 0)
$$

is bounded away from 0 and from $\infty$. More precisely,

$$
1 \leq \Theta_{n} \leq \frac{u_{1}}{u_{2}}\left(\frac{\left\|W_{\alpha}\right\|^{2}}{\alpha_{0} \alpha_{1}}\right)^{2} \quad \text { for sufficiently large } n .
$$

In particular, $\left\{u_{n}\right\}_{n=0}^{\infty}$ is eventually decreasing.

Proof of Theorem 2.3. By Theorem 2.2, $W_{\alpha}$ is strictly positively quadratically hyponormal, in the sense that all coefficients of $d_{n}(t)$ are positive for all $n \geq 0$. Note that finite rank perturbations of $\alpha$ affect a finite number of values of $u_{n}, v_{n}$ and $w_{n}$. More concretely, if $\alpha^{\prime}$ is a perturbation of $\alpha$ in the weights $\left\{\alpha_{0}, \cdots, \alpha_{N}\right\}$, then $u_{n}, v_{n}, w_{n}$ and $p_{n}$ are invariant under $\alpha^{\prime}$ for $n \geq N+3$. In particular, $p_{n} \geq 0$ for $n \geq N+3$.

Claim 1. For $n \geq 3,0 \leq i \leq n+1$,

$$
\begin{aligned}
c(n, i)= & u_{n} c(n-1, i)+p_{n-1} c(n-2, i-1) \\
& +\sum_{k=4}^{n} p_{k-2}\left(\prod_{j=k}^{n} v_{j}\right) c(k-3, i-n+k-2)+v_{n} \cdots v_{3} \rho_{i-n+1},
\end{aligned}
$$


where

$$
\rho_{i-n+1}= \begin{cases}0 & (i<n-1), \\ u_{0} p_{1} & (i=n-1), \\ v_{0} p_{1}+v_{2} p_{0} & (i=n), \\ v_{0} v_{1} v_{2} & (i=n+1)\end{cases}
$$

(cf. [12, Proof of Theorem 4.3]).

Proof of Claim 1. We use induction. For $n=3,0 \leq i \leq 4$,

$$
\begin{aligned}
c(3, i)= & u_{3} c(2, i)+v_{3} c(2, i-1)-w_{2} c(1, i-1) \\
= & u_{3} c(2, i)+v_{3}\left(u_{2} c(1, i-1)+v_{2} c(1, i-2)-w_{1} c(0, i-2)\right) \\
& -w_{2} c(1, i-1) \\
= & u_{3} c(2, i)+p_{2} c(1, i-1)+v_{3}\left(v_{2} c(1, i-2)-w_{1} c(0, i-2)\right) \\
= & u_{3} c(2, i)+p_{2} c(1, i-1)+v_{3} \rho_{i-2},
\end{aligned}
$$

where by (4.3),

$$
\rho_{i-2}= \begin{cases}0 & (i<2) \\ u_{0} p_{1} & (i=2) \\ v_{0} p_{1}+v_{2} p_{0} & (i=3) \\ v_{0} v_{1} v_{2} & (i=4)\end{cases}
$$

Now,

$$
\begin{aligned}
c(n+1, i)= & u_{n+1} c(n, i)+v_{n+1} c(n, i-1)-w_{n} c(n-1, i-1) \\
= & u_{n+1} c(n, i)+v_{n+1}\left(u_{n} c(n-1, i-1)+p_{n-1} c(n-2, i-2)\right. \\
& \left.+\sum_{k=4}^{n} p_{k-2}\left(\prod_{j=k}^{n} v_{j}\right) c(k-3, i-n+k-3)+v_{n} \cdots v_{3} \rho_{i-n}\right) \\
& -w_{n} c(n-1, i-1) \\
= & u_{n+1} c(n, i)+p_{n} c(n-1, i-1)+v_{n+1} p_{n-1} c(n-2, i-2) \\
& +v_{n+1} \sum_{k=4}^{n} p_{k-2}\left(\prod_{j=k}^{n} v_{j}\right) c(k-3, i-n+k-3)+v_{n+1} \cdots v_{3} \rho_{i-n} \\
& (\text { by the inductive hypothesis }) \\
= & u_{n+1} c(n, i)+p_{n} c(n-1, i-1) \\
& +\sum_{k=4}^{n+1} p_{k-2}\left(\prod_{j=k}^{n+1} v_{j}\right) c(k-3, i-n+k-3)+v_{n+1} \cdots v_{3} \rho_{i-n},
\end{aligned}
$$

which proves Claim 1. 
Write $u_{n}^{\prime}, v_{n}^{\prime}, w_{n}^{\prime}, p_{n}^{\prime}, \rho_{n}^{\prime}$, and $c^{\prime}(\cdot, \cdot)$ for the entities corresponding to $\alpha^{\prime}$. If $p_{n}>$ 0 for every $n=0, \cdots, N+2$, then in view of Claim 1, we can choose a small perturbation such that $p_{n}^{\prime}>0(0 \leq n \leq N+2)$ and therefore $c^{\prime}(n, i)>0$ for all $n \geq 0$ and $0 \leq i \leq n+1$, which implies that $W_{\alpha^{\prime}}$ is also positively quadratically hyponormal. If instead $p_{n}=0$ for some $n=0, \cdots, N+2$, careful inspection of (5.3) reveals that without loss of generality we may assume $p_{0}=\cdots=p_{N+2}=0$. By Theorem 2.2, we have that for a sufficiently small perturbation $\alpha^{\prime}$ of $\alpha$,

$$
c^{\prime}(n, i)>0 \quad(0 \leq n \leq N+2,0 \leq i \leq n+1) \quad \text { and } \quad c^{\prime}(n, n+1)>0 \quad(n \geq 0) .
$$

Write

$$
k_{n}:=\frac{v_{n}}{u_{n}} \quad(n=2,3, \cdots) .
$$

Claim 2. $\left\{k_{n}\right\}_{n=2}^{\infty}$ is bounded.

Proof of Claim 2. Observe that

$$
\begin{aligned}
k_{n}=\frac{v_{n}}{u_{n}} & =\frac{\alpha_{n}^{2} \alpha_{n+1}^{2}-\alpha_{n-1}^{2} \alpha_{n-2}^{2}}{\alpha_{n}^{2}-\alpha_{n-1}^{2}} \\
& =\alpha_{n}^{2}+\alpha_{n-1}^{2}+\alpha_{n}^{2} \frac{\alpha_{n+1}^{2}-\alpha_{n}^{2}}{\alpha_{n}^{2}-\alpha_{n-1}^{2}}+\alpha_{n-1}^{2} \frac{\alpha_{n-1}^{2}-\alpha_{n-2}^{2}}{\alpha_{n}^{2}-\alpha_{n-1}^{2}} .
\end{aligned}
$$

Therefore if $W_{\alpha}$ is 2-hyponormal, then by Lemma 5.1, the sequences

$$
\left\{\frac{\alpha_{n+1}^{2}-\alpha_{n}^{2}}{\alpha_{n}^{2}-\alpha_{n-1}^{2}}\right\}_{n=2}^{\infty} \text { and } \quad\left\{\frac{\alpha_{n-1}^{2}-\alpha_{n-2}^{2}}{\alpha_{n}^{2}-\alpha_{n-1}^{2}}\right\}_{n=2}^{\infty}
$$

are both bounded, so that $\left\{k_{n}\right\}_{n=2}^{\infty}$ is bounded. This proves Claim 2 .

Write $k:=\sup _{n} k_{n}$. Without loss of generality we assume $k<1$ (this is possible from the observation that $c \alpha$ induces $\left\{c^{2} k_{n}\right\}$ ). Choose a sufficiently small perturbation $\alpha^{\prime}$ of $\alpha$ such that if we let

$$
h:=\sup _{\substack{0 \leq \ell \leq N+2 \\ 0 \leq m \leq 1}}\left|\sum_{k=4}^{N+4} p_{k-2}^{\prime}\left(\prod_{j=k}^{N+3} v_{j}^{\prime}\right) c^{\prime}(k-3, \ell)+v_{N+3}^{\prime} \cdots v_{3}^{\prime} \rho_{m}^{\prime}\right|,
$$

then

$$
c^{\prime}(N+3, i)-\frac{1}{1-k} h>0 \quad(0 \leq i \leq N+3)
$$

(this is always possible because, by Theorem 2.2, we can choose a sufficiently small $\left|p_{i}^{\prime}\right|$ such that

$c^{\prime}(N+3, i)>v_{0} \cdots v_{i-1} u_{i} \cdots u_{N+3}-\epsilon \quad$ and $\quad|h|<(1-k)\left(v_{0} \cdots v_{i-1} u_{i} \cdots u_{N+3}-\epsilon\right)$

for any small $\epsilon>0)$. 
Claim 3. For $j \geq 4$ and $0 \leq i \leq N+j$,

$$
c^{\prime}(N+j, i) \geq u_{N+j} \cdots u_{N+4}\left(c^{\prime}(N+3, i)-\sum_{n=1}^{j-3} k^{n} h\right) .
$$

Proof of Claim 3. We use induction. If $j=4$, then by Claim 1 and (5.6),

$$
\begin{aligned}
c^{\prime}(N+4, i)= & u_{N+4}^{\prime} c^{\prime}(N+3, i)+p_{N+3}^{\prime} c^{\prime}(N+2, i-1) \\
& +v_{N+4}^{\prime} \sum_{k=4}^{N+4} p_{k-2}^{\prime}\left(\prod_{j=k}^{N+3} v_{j}^{\prime}\right) c^{\prime}(k-3, i-N+k-6) \\
& +v_{N+4}^{\prime} \cdots v_{3}^{\prime} \rho_{i-(N+3)}^{\prime} \\
\geq & u_{N+4}^{\prime} c^{\prime}(N+3, i)+p_{N+3}^{\prime} c^{\prime}(N+2, i-1)-v_{N+4}^{\prime} h \\
\geq & u_{N+4}\left(c^{\prime}(N+3, i)-k_{N+4} h\right) \\
\geq & u_{N+4}\left(c^{\prime}(N+3, i)-k h\right),
\end{aligned}
$$

because $u_{N+4}^{\prime}=u_{N+4}, v_{N+4}^{\prime}=v_{N+4}$ and $p_{N+3}^{\prime}=p_{N+3} \geq 0$. Now suppose (5.8) holds for some $j \geq 4$. By Claim 1 , we have that for $j \geq 4$,

$$
\begin{aligned}
c^{\prime}(N+j+1, i)= & u_{N+j+1}^{\prime} c^{\prime}(N+j, i)+p_{N+j}^{\prime} c(N+j-1, i-1) \\
& +\sum_{k=4}^{N+j+1} p_{k-2}^{\prime}\left(\prod_{j=k}^{N+j+1} v_{j}^{\prime}\right) c^{\prime}(k-3, i-N+k-j-3) \\
& +v_{N+j+1}^{\prime} \cdots v_{3}^{\prime} \rho_{i-(N+j)}^{\prime} \\
= & u_{N+j+1}^{\prime} c^{\prime}(N+j, i)+p_{N+j}^{\prime} c(N+j-1, i-1) \\
& +\sum_{k=N+5}^{N+j+1} p_{k-2}^{\prime}\left(\prod_{j=k}^{N+j+1} v_{j}^{\prime}\right) c^{\prime}(k-3, i-N+k-j-3) \\
& +\sum_{k=4}^{N+4} p_{k-2}^{\prime}\left(\prod_{j=k}^{N+j+1} v_{j}^{\prime}\right) c^{\prime}(k-3, i-N+k-j-3) \\
& +v_{N+j+1}^{\prime} \cdots v_{3}^{\prime} \rho_{i-(N+j)}^{\prime} .
\end{aligned}
$$

Since $p_{n}^{\prime}=p_{n}>0$ for $n \geq N+3$ and $c^{\prime}(n, \ell)>0$ for $0 \leq n \leq N+j$ by the inductive hypothesis, it follows that

$$
p_{N+j}^{\prime} c(N+j-1, i-1)+\sum_{k=N+5}^{N+j+1} p_{k-2}^{\prime}\left(\prod_{j=k}^{N+j+1} v_{j}^{\prime}\right) c^{\prime}(k-3, i-N+k-j-3) \geq 0
$$


By the inductive hypothesis and (5.9),

$$
\begin{aligned}
& c^{\prime}(N+j+1, i) \\
& \geq u_{N+j+1}^{\prime} c^{\prime}(N+j, i)+\sum_{k=4}^{N+4} p_{k-2}^{\prime}\left(\prod_{j=k}^{N+j+1} v_{j}^{\prime}\right) c^{\prime}(k-3, i-N+k-j-3) \\
& \quad+v_{N+j+1}^{\prime} \cdots v_{3}^{\prime} \rho_{i-(N+j)}^{\prime} \\
& \geq u_{N+j+1} u_{N+j} \cdots u_{N+4}\left(c^{\prime}(N+3, i)-\sum_{n=1}^{j-3} k^{n} h\right) \\
& \quad+v_{N+j+1} v_{N+j} \cdots v_{N+4}\left(\sum_{k=4}^{N+4} p_{k-2}^{\prime}\left(\prod_{j=k}^{N+3} v_{j}^{\prime}\right) c^{\prime}(k-3, i-N+k-j-3)\right. \\
& \geq u_{N+j+1} u_{N+j} \cdots u_{N+4}\left(c^{\prime}(N+3, i)-\sum_{n=1}^{j-3} k^{n} h\right)-v_{N+j+1} v_{N+j} \cdots v_{N+4} h \\
& =u_{N+j+1} u_{N+j} \cdots u_{N+4}\left(c^{\prime}(N+3, i)-\sum_{n=1}^{j-3} k^{n} h-k_{N+j+1} k_{N+j} \cdots k_{N+4} h\right) \\
& \geq u_{N+j+1}^{\prime} u_{N+j}^{\prime} \cdots u_{N+4}^{\prime}\left(c^{\prime}(N+3, i)-\sum_{n=1}^{j-2} k^{n} h\right),
\end{aligned}
$$

which proves Claim 3.

Since $\sum_{n=1}^{j} k^{n}<\frac{1}{1-k}$ for every $j>1$, it follows from Claim 3 and (5.7) that

$$
c^{\prime}(N+j, i)>0 \text { for } j \geq 4 \text { and } 0 \leq i \leq N+j .
$$

It thus follows from (5.4) and (5.10) that $c^{\prime}(n, i)>0$ for every $n \geq 0$ and $0 \leq i \leq$ $n+1$. Therefore $W_{\alpha^{\prime}}$ is also positively quadratically hyponormal. This completes the proof.

Corollary 5.2. Let $W_{\alpha}$ be a weighted shift such that $\alpha_{j-1}<\alpha_{j}$ for some $j \geq 1$, and let $T_{x}$ be the weighted shift with weight sequence

$$
\alpha_{x}: \alpha_{0}, \cdots, \alpha_{j-1}, x, \alpha_{j+1}, \cdots .
$$

Then $\left\{x: T_{x}\right.$ is 2-hyponormal $\}$ is a proper closed subset of $\left\{x: T_{x}\right.$ is quadratically hyponormal\} whenever the latter set is nonempty.

Proof. Write

$$
H_{2}:=\left\{x: T_{x} \text { is 2-hyponormal }\right\} .
$$

Without loss of generality, we can assume that $H_{2}$ is nonempty, and that $j=1$. Recall that a 2-hyponormal weighted shift with two equal weights is of the form $\alpha_{0}=\alpha_{1}=\alpha_{2}=\cdots$ or $\alpha_{0}<\alpha_{1}=\alpha_{2}=\alpha_{3}=\cdots$. Let $x_{m}:=\inf H_{2}$. By Proposition 6.7 below, $T_{x_{m}}$ is hyponormal. Then $x_{m}>\alpha_{0}$. By assumption, $x_{m}<$ $\alpha_{2}$. Thus $\alpha_{0}, x_{m}, \alpha_{2}, \alpha_{3}, \cdots$ is strictly increasing. Now we apply Theorem 2.3 to obtain $x^{\prime}$ such that $\alpha_{0}<x^{\prime}<x_{m}$ and $T_{x^{\prime}}$ is quadratically hyponormal. However $T_{x^{\prime}}$ is not 2-hyponormal by the definition of $x_{m}$. The proof is complete. 
The following question arises naturally:

Question 5.3. Let $\alpha$ be a strictly increasing weight sequence and let $k \geq 3$. If $W_{\alpha}$ is a $k$-hyponormal weighted shift, does it follow that $W_{\alpha}$ is weakly $k$-hyponormal under a small perturbation of the weight sequence?

\section{Other Related Results}

6.1. Subnormal extensions. Let $\alpha: \alpha_{0}, \alpha_{1}, \cdots$ be a weight sequence, let $x_{i}>0$ for $1 \leq i \leq n$, and let $\left(x_{n}, \cdots, x_{1}\right) \alpha: x_{n}, \cdots, x_{1}, \alpha_{0}, \alpha_{1}, \cdots$ be the augmented weight sequence. We say that $W_{\left(x_{n}, \cdots, x_{1}\right) \alpha}$ is an extension (or $n$-step extension) of $W_{\alpha}$. Observe that

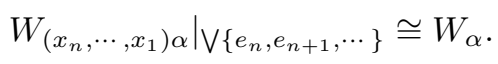

The hypothesis $F \neq c P_{\left\{e_{0}\right\}}$ in Theorem 2.1 is essential. Indeed, there exist infinitely many one-step subnormal extensions of a subnormal weighted shift whenever one such extension exists. Recall ([7, Proposition 8]) that if $W_{\alpha}$ is a weighted shift whose restriction to $\bigvee\left\{e_{1}, e_{2}, \cdots\right\}$ is subnormal with associated measure $\mu$, then $W_{\alpha}$ is subnormal if and only if

(i) $\frac{1}{t} \in L^{1}(\mu)$;

(ii) $\alpha_{0}^{2} \leq\left(\left\|\frac{1}{t}\right\|_{L^{1}(\mu)}\right)^{-1}$.

Also note that there may not exist any one-step subnormal extension of the subnormal weighted shift: for example, if $W_{\alpha}$ is the Bergman shift, then the corresponding Berger measure is $\mu(t)=t$, and hence $\frac{1}{t}$ is not integrable with respect to $\mu$; therefore $W_{\alpha}$ does not admit any subnormal extension. A similar situation arises when $\mu$ has an atom at $\{0\}$.

More generally we have:

Theorem 6.1 (Subnormal Extensions). Let $W_{\alpha}$ be a subnormal weighted shift with weights $\alpha: \alpha_{0}, \alpha_{1}, \cdots$ and let $\mu$ be the corresponding Berger measure. Then $W_{\left(x_{n}, \cdots, x_{1}\right) \alpha}$ is subnormal if and only if

(i) $\frac{1}{t^{n}} \in L^{1}(\mu)$;

(ii) $x_{j}=\left(\frac{\left\|\frac{1}{t^{j}-I}\right\|_{L^{1}(\mu)}}{\left\|\frac{1}{t^{j}}\right\|_{L^{1}(\mu)}}\right)^{\frac{1}{2}}$ for $1 \leq j \leq n-1$;

(iii) $x_{n} \leq\left(\frac{\left\|\frac{1}{t^{n-1}}\right\|_{L^{1}(\mu)}}{\left\|\frac{1}{t^{n}}\right\|_{L^{1}(\mu)}}\right)^{\frac{1}{2}}$.

In particular, if we put

$$
S:=\left\{\left(x_{1}, \cdots, x_{n}\right) \in \mathbb{R}^{n}: W_{\left(x_{n}, \cdots, x_{1}\right) \alpha} \text { is subnormal }\right\},
$$

then either $S=\emptyset$ or $S$ is a line segment in $\mathbb{R}^{n}$.

Proof. Write $W_{j}:=W_{\left(x_{n}, \cdots, x_{1}\right) \alpha} \mid \bigvee\left\{e_{n-j}, e_{n-j+1}, \cdots\right\}(1 \leq j \leq n)$ and hence $W_{n}=$ $W_{\left(x_{n}, \cdots, x_{1}\right) \alpha}$. By the argument used to establish (3.2) we have that $W_{1}$ is subnormal with associated measure $\nu_{1}$ if and only if

(i) $\frac{1}{t} \in L^{1}(\mu)$;

(ii) $d \nu_{1}=\frac{x_{1}^{2}}{t} d \mu$, or equivalently, $x_{1}^{2}=\left(\int_{0}^{\left\|W_{\alpha}\right\|^{2}} \frac{1}{t} d \mu(t)\right)^{-1}$.

Inductively $W_{n-1}$ is subnormal with associated measure $\nu_{n-1}$ if and only if

(i) $W_{n-2}$ is subnormal;

(ii) $\frac{1}{t^{n-1}} \in L^{1}(\mu)$; 
(iii) $d \nu_{n-1}=\frac{x_{n-1}^{2}}{t} d \nu_{n-2}=\cdots=\frac{x_{n-1}^{2} \cdots x_{1}^{2}}{t^{n-1}} d \mu$, or equivalently,

$$
x_{n-1}^{2}=\frac{\int_{0}^{\left\|W_{\alpha}\right\|^{2}} \frac{1}{t^{n-2}} d \mu(t)}{\int_{0}^{\left\|W_{\alpha}\right\|^{2}} \frac{1}{t^{n-1}} d \mu(t)} .
$$

Therefore $W_{n}$ is subnormal if and only if

(i) $W_{n-1}$ is subnormal;

(ii) $\frac{1}{t^{n}} \in L^{1}(\mu)$;

(iii) $x_{n}^{2} \leq\left(\int_{0}^{\left\|W_{\alpha}\right\|^{2}} \frac{1}{t} d \nu_{n-1}\right)^{-1}=\left(\int_{0}^{\left\|W_{\alpha}\right\|^{2}} \frac{x_{n-1}^{2} \cdots x_{1}^{2}}{t^{n}} d \mu(t)\right)^{-1}$

$$
=\frac{\int_{0}^{\left\|W_{\alpha}\right\|^{2}} \frac{1}{t^{n-1}} d \mu(t)}{\int_{0}^{\left\|W_{\alpha}\right\|^{2}} \frac{1}{t^{n}} d \mu(t)} \text {. }
$$

Corollary 6.2. If $W_{\alpha}$ is a subnormal weighted shift with associated measure $\mu$, there exists an n-step subnormal extension of $W_{\alpha}$ if and only if $\frac{1}{t^{n}} \in L^{1}(\mu)$.

For the next result we refer to the notation in (2.1) and (2.2).

Corollary 6.3. A recursively generated subnormal shift with $\varphi_{0} \neq 0$ admits an $n$-step subnormal extension for every $n \geq 1$.

Proof. The assumption about $\varphi_{0}$ implies that the zeros of $g(t)$ are positive, so that $s_{0}>0$. Thus for every $n \geq 1, \frac{1}{t^{n}}$ is integrable with respect to the corresponding Berger measure $\mu=\rho_{0} \delta_{s_{0}}+\cdots+\rho_{r-1} \delta_{s_{r-1}}$. By Corollary 6.2, there exists an $n$-step subnormal extension.

We need not expect that for arbitrary recursively generated shifts, 2-hyponormality and subnormality coincide as in Theorem 3.2. For example, if $\alpha: \sqrt{\frac{1}{2}}, \sqrt{x}$, $\left(\sqrt{3}, \sqrt{\frac{10}{3}}, \sqrt{\frac{17}{5}}\right)^{\wedge}$, then by $(2.12)$ and Theorem 6.1 ,

(i) $T_{x}$ is 2-hyponormal $\Longleftrightarrow 4-\sqrt{6} \leq x \leq 2$;

(ii) $T_{x}$ is subnormal $\Longleftrightarrow x=2$.

A straightforward calculation shows, however, that $T_{x}$ is 3-hyponormal if and only if $x=2$; for,

$$
A(0 ; 3):=\left(\begin{array}{cccc}
1 & \frac{1}{2} & \frac{1}{2} x & \frac{3}{2} x \\
\frac{1}{2} & \frac{1}{2} x & \frac{3}{2} x & 5 x \\
\frac{1}{2} x & \frac{3}{2} x & 5 x & 17 x \\
\frac{3}{2} x & 5 x & 17 x & 58 x
\end{array}\right) \geq 0 \Longleftrightarrow x=2 .
$$

This behavior is typical of general recursively generated weighted shifts: we show in [13] that subnormality is equivalent to $k$-hyponormality for some $k \geq 2$.

6.2. Convexity and closedness. Next, we will show that canonical rank-one perturbations of $k$-hyponormal weighted shifts which preserve $k$-hyponormality form a convex set. To see this we need an auxiliary result.

Lemma 6.4. Let $I=\{1, \cdots, n\} \times\{1, \cdots, n\}$ and let $J$ be a symmetric subset of I. Let $A=\left(a_{i j}\right) \in M_{n}(\mathbb{C})$ and let $C=\left(c_{i j}\right) \in M_{n}(\mathbb{C})$ be given by

$$
c_{i j}=\left\{\begin{array}{ll}
c a_{i j} & \text { if }(i, j) \in J \\
a_{i j} & \text { if }(i, j) \in I \backslash J
\end{array} \quad(c>0) .\right.
$$


If $A$ and $C$ are positive semidefinite, then $B=\left(b_{i j}\right) \in M_{n}(\mathbb{C})$ defined by

$$
b_{i j}=\left\{\begin{array}{ll}
b a_{i j} & \text { if }(i, j) \in J \\
a_{i j} & \text { if }(i, j) \in I \backslash J
\end{array} \quad(b \in[1, c] \text { or }[c, 1])\right.
$$

is also positive semidefinite.

Proof. Without loss of generality we may assume $c>1$. If $b=1$ or $b=c$, the assertion is trivial. Thus we assume $1<b<c$. The result is now a consequence of the following observation. If $[D]_{(i, j)}$ denotes the $(i, j)$-entry of the matrix $D$, then

$$
\begin{aligned}
{\left[\frac{c-b}{c-1}\left(A+\frac{b-1}{c-b} C\right)\right]_{(i, j)} } & = \begin{cases}\frac{c-b}{c-1}\left(1+\frac{b-1}{c-b} c\right) a_{i j} & \text { if }(i, j) \in J, \\
\frac{c-b}{c-1}\left(1+\frac{b-1}{c-b}\right) a_{i j} & \text { if }(i, j) \in I \backslash J,\end{cases} \\
& = \begin{cases}b a_{i j} & \text { if }(i, j) \in J, \\
a_{i j} & \text { if }(i, j) \in I \backslash J,\end{cases} \\
& =[B]_{(i, j)},
\end{aligned}
$$

which is positive semidefinite because positive semidefinite matrices in $M_{n}(\mathbb{C})$ form a cone.

An immediate consequence of Lemma 6.4 is that positivity of a matrix forms a convex set with respect to a fixed diagonal location; i.e., if

$$
A_{x}=\left(\begin{array}{lll}
* & * & * \\
& x & * \\
* & * & *
\end{array}\right),
$$

then $\left\{x: A_{x}\right.$ is positive semidefinite $\}$ is convex.

We now have:

Theorem 6.5. Let $\alpha=\left\{\alpha_{n}\right\}_{n=0}^{\infty}$ be a weight sequence, let $k \geq 1$, and let $j \geq 0$. Define $\alpha^{(j)}(x): \alpha_{0}, \cdots, \alpha_{j-1}, x, \alpha_{j+1}, \cdots$. Assume $W_{\alpha}$ is $k$-hyponormal and define

$$
\Omega_{\alpha}^{k, j}:=\left\{x: W_{\alpha^{(j)}(x)} \text { is k-hyponormal }\right\} .
$$

Then $\Omega_{\alpha}^{k, j}$ is a closed interval.

Proof. Suppose $x_{1}, x_{2} \in \Omega_{\alpha}^{k, j}$ with $x_{1}<x_{2}$. Then by $(2.11)$, the $(k+1) \times(k+1)$ Hankel matrix

$$
A_{x_{i}}(n ; k):=\left(\begin{array}{cccc}
\gamma_{n} & \gamma_{n+1} & \ldots & \gamma_{n+k} \\
\gamma_{n+1} & \gamma_{n+2} & \ldots & \gamma_{n+k+1} \\
\vdots & \vdots & & \vdots \\
\gamma_{n+k} & \gamma_{n+k+1} & \ldots & \gamma_{n+2 k}
\end{array}\right) \quad(n \geq 0 ; i=1,2)
$$

is positive, where $A_{x_{i}}$ corresponds to $\alpha^{(j)}\left(x_{i}\right)$. We must show that $t x_{1}+(1-t) x_{2} \in$ $\Omega_{\alpha}^{k, j}(0<t<1)$, i.e.,

$$
A_{t x_{1}+(1-t) x_{2}}(n ; k) \geq 0 \quad(n \geq 0,0<t<1) .
$$

Observe that it suffices to establish the positivity of the $2 k$ Hankel matrices corresponding to $\alpha^{(j)}\left(t x_{1}+(1-t) x_{2}\right)$ such that $t x_{1}+(1-t) x_{2}$ appears as a factor in at least one entry but not in every entry. A moment's thought reveals that without loss of generality we may assume $j=2 k$. Observe that

$$
A_{z_{1}}(n ; k)-A_{z_{2}}(n ; k)=\left(z_{1}^{2}-z_{2}^{2}\right) H(n ; k)
$$


for some Hankel matrix $H(n ; k)$. For notational convenience, we abbreviate $A_{z}(n ; k)$ as $A_{z}$. Then

$$
A_{t x_{1}+(1-t) x_{2}}= \begin{cases}t^{2} A_{x_{1}}+(1-t)^{2} A_{x_{2}}+2 t(1-t) A_{\sqrt{x_{1} x_{2}}} & \text { for } 0 \leq n \leq 2 k, \\ \left(t+(1-t) \frac{x_{2}}{x_{1}}\right)^{2} A_{x_{1}} & \text { for } n \geq 2 k+1 .\end{cases}
$$

Since $A_{x_{1}} \geq 0, A_{x_{2}} \geq 0$ and $A_{\sqrt{x_{1} x_{2}}}$ have the form described by Lemma 6.4 and since $x_{1}<\sqrt{x_{1} x_{2}}<x_{2}$, it follows from Lemma 6.4 that $A_{\sqrt{x_{1} x_{2}}} \geq 0$. Thus evidently, $A_{t x_{1}+(1-t) x_{2}} \geq 0$, and therefore $t x_{1}+(1-t) x_{2} \in \Omega_{\alpha}^{k, j}$. This shows that $\Omega_{\alpha}^{k, j}$ is an interval. The closedness of the interval follows from Proposition 6.7 below.

In [17] and [18, it was shown that there exists a nonsubnormal polynomially hyponormal operator. Also in 22 , it was shown that there exists a nonsubnormal polynomially hyponormal operator if and only if there exists one which is also a weighted shift. However, no concrete weighted shift has yet been found. As a strategy for finding such a shift, we would like to suggest the following:

Question 6.6. Does it follow that the polynomial hyponormality of a weighted shift is stable under small perturbations of the weight sequence?

If the answer to Question 6.6 were affirmative then we would easily find a polynomially hyponormal nonsubnormal (even non-2-hyponormal) weighted shift; for example, if

$$
\alpha: 1, \sqrt{x},\left(\sqrt{3}, \sqrt{\frac{10}{3}}, \sqrt{\frac{17}{5}}\right)^{\wedge}
$$

and $T_{x}$ is the weighted shift associated with $\alpha$, then by Theorem 3.2, $T_{x}$ is subnormal $\Leftrightarrow x=2$, whereas $T_{x}$ is polynomially hyponormal $\Leftrightarrow 2-\delta_{1}<x<2+\delta_{2}$ for some $\delta_{1}, \delta_{2}>0$ provided the answer to Question 6.6 is yes; therefore for sufficiently small $\epsilon>0$,

$$
\alpha_{\epsilon}: 1, \sqrt{2+\epsilon},\left(\sqrt{3}, \sqrt{\frac{10}{3}}, \sqrt{\frac{17}{5}}\right)^{\wedge}
$$

would induce a non-2-hyponormal polynomially hyponormal weighted shift.

The answer to Question 6.6 for weak $k$-hyponormality is negative. In fact we have:

Proposition 6.7. (i) The set of k-hyponormal operators is sot-closed.

(ii) The set of weakly $k$-hyponormal operators is sot-closed.

Proof. Suppose $T_{\eta} \in \mathcal{L}(\mathcal{H})$ and $T_{\eta} \rightarrow T$ in sot. Then, by the Uniform Boundedness Principle, $\left\{\left\|T_{\eta}\right\|\right\}_{\eta}$ is bounded. Thus $T_{\eta}^{* i} T_{\eta}^{j} \rightarrow T^{* i} T^{j}$ in sot for every $i, j$, so that $M_{k}\left(T_{\eta}\right) \rightarrow M_{k}(T)$ in sot (where $M_{k}(T)$ is as in (1.2)).

(i) In this case $M_{k}\left(T_{\eta}\right) \geq 0$ for all $\eta$, so $M_{k}(T) \geq 0$, i.e., $T$ is $k$-hyponormal.

(ii) Here, $M_{k}\left(T_{\eta}\right)$ is weakly positive for all $\eta$. By $(1.3), M_{k}(T)$ is also weakly positive, i.e., $T$ is weakly $k$-hyponormal.

\section{REFERENCES}

1. A. Athavale, On joint hyponormality of operators, Proc. Amer. Math. Soc. 103 (1988), 417423. MR0943059 (89f:47033)

2. J. Bram, Subnormal operators, Duke Math. J. 22 (1955), 75-94. MR0068129 (16:835a)

3. Y.B. Choi, J.K. Han and W.Y. Lee, One-step extension of the Bergman shift, Proc. Amer. Math. Soc. 128 (2000), 3639-3646. MR1694855 (2001b:47037) 
4. J.B. Conway, The Theory of Subnormal Operators, Math. Surveys and Monographs, vol. 36, Amer. Math. Soc., Providence, 1991. MR1112128 (92h:47026)

5. J.B. Conway and W. Szymanski, Linear combination of hyponormal operators, Rocky Mountain J. Math. 18 (1988), 695-705. MR0972659 (90a:47059)

6. C. Cowen, Hyponormal and subnormal Toeplitz operators, Surveys of Some Recent Results in Operator Theory, I (J.B. Conway and B.B. Morrel, eds.), Pitman Research Notes in Mathematics, Vol. 171, Longman, 1988, pp. 155-167. MR0958573 (90j:47022)

7. R.E. Curto, Quadratically hyponormal weighted shifts, Integral Equations Operator Theory 13 (1990), 49-66. MR1025673 (90k:47061)

8. __ Joint hyponormality: A bridge between hyponormality and subnormality, Proc. Sympos. Pure Math., vol. 51, Part 2, Amer. Math. Soc., Providence, 1990, pp. 69-91. MR1077422 (91k:47049)

9. Banach Center Publications 38 (1997), 75-104. MR1457002 (99c:47014)

10. R.E. Curto and L.A. Fialkow, Recursiveness, positivity, and truncated moment problems, Houston J. Math. 17 (1991), 603-635. MR.1147276 (93a:47016)

11. _ Recursively generated weighted shifts and the subnormal completion problem, Integral Equations Operator Theory 17 (1993), 202-246. MR1233668 (94h:47050)

12. — Recursively generated weighted shifts and the subnormal completion problem, II, Integral Equations Operator Theory 18 (1994), 369-426. MR.1265443 (94m:47044)

13. R.E. Curto, I.B. Jung and W.Y. Lee, Extensions and extremality of recursively generated weighted shifts, Proc. Amer. Math. Soc. 130 (2002), 565-576. MR1862138 (2002i:47041)

14. R.E. Curto and W.Y. Lee, Joint hyponormality of Toeplitz pairs, Mem. Amer. Math. Soc. no. 712, Amer. Math. Soc., Providence, 2001. MR1810770 (2002c:47042)

15. - Towards a model theory for 2-hyponormal operators, Integral Equations Operator Theory 44 (2002), 290-315. MR1933654 (2003m:47036)

16. R.E. Curto, P.S. Muhly and J. Xia, Hyponormal pairs of commuting operators, Contributions to Operator Theory and Its Applications (Mesa, AZ, 1987) (I. Gohberg, J.W. Helton and L. Rodman, eds.), Operator Theory: Advances and Applications, vol. 35, Birkhäuser, BaselBoston, 1988, pp. 1-22. MR.1017663 (90m:47037)

17. R.E. Curto and M. Putinar, Existence of non-subnormal polynomially hyponormal operators, Bull. Amer. Math. Soc. (N.S.) 25 (1991), 373-378. MR1091568 (93e:47028)

18. _ Nearly subnormal operators and moment problems, J. Funct. Anal. 115 (1993), 480497. MR.1234402 (95d:47024)

19. R.G. Douglas, V.I. Paulsen, and K. Yan, Operator theory and algebraic geometry, Bull. Amer. Math. Soc. (N.S.) 20 (1989), 67-71. MR0955316 (90f:47028)

20. P.R. Halmos, Ten problems in Hilbert space, Bull. Amer. Math. Soc. 76 (1970), 887-933. MR 0270173 (42:5066)

21. , A Hilbert Space Problem Book, 2nd ed., Springer, New York, 1982. MR 0675952 (84e:47001)

22. S. McCullough and V. Paulsen, A note on joint hyponormality, Proc. Amer. Math. Soc. 107 (1989), 187-195. MR0972236 (90a:47062)

23. A. Shields, Weighted shift operators and analytic function theory, Math. Surveys 13 (1974), 49-128. MR0361899(50:14341)

Department of Mathematics, University of Iowa, Iowa City, Iowa 52242

E-mail address: rcurto@math.uiowa.edu

Department of Mathematics, Seoul National University, Seoul 151-742, Korea

E-mail address: wylee@math.snu.ac.kr 